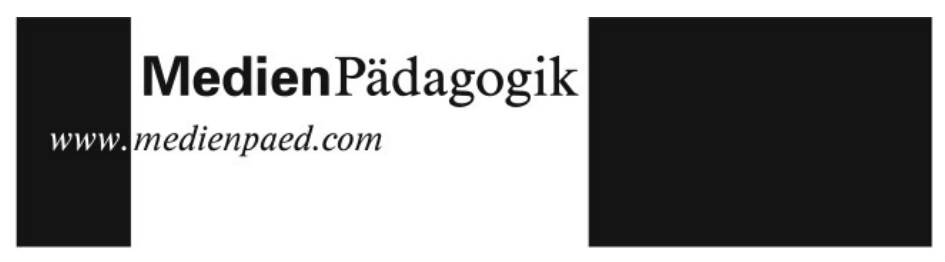

Rezensionen

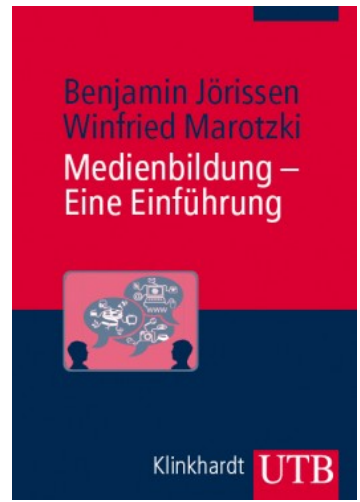

Benjamin Jörissen und Winfried Marotzki

Medienbildung - Eine Einführung

Stuttgart: UTB, 2009. 260 Seiten

ISBN-13: 978-3825231897

$€ 18,90 ;$ CHF 32,90

\title{
Medienbildung als Umgang mit gesellschaftlicher Kontingenz in und mithilfe von Medien
}

Mit ihrer Monographie «Medienbildung - Eine Einführung» legen Benjamin Jörissen und Winfried Marotzki einen wichtigen Diskussionsbeitrag zur theoretisch-konzeptionellen Standortbestimmung der Medienpädagogik vor. Obwohl es seit Ende der 1990er Jahre verstärkt Versuche gibt, Medienbildung grundlegend im Selbstverständnis der Disziplin zu etablieren, ist es bisher kaum gelungen, einen - jenseits der bloßen Nennung des Begriffs in bildungspolitischen Positionspapieren, auf Tagungen und in Titeln diverser wissenschaftlicher wie nicht-wissenschaftlicher Aufsätze und Bände - auch tiefergehenden, systematischen Begründungsrahmen vorzulegen. Jörissen/ Marotzki ist dies ohne Zweifel gelungen.

Inhaltlich gliedert sich das Buch in zwei Teile. Der erste Teil des Buches bietet eine grundlegende theoretische Herleitung einer Strukturalen Medienbildung aus vornehmlich bildungstheoretischer Perspektive (Kapitel 2), aufbauend auf der von Marotzki (1990) entworfenen strukturalen Bildungstheorie. Der zweite Teil beinhaltet daran anknüpfend eine Analyse von Bildungsprozessen in Film, Bild und Internet (Kapitel 3-5).

Im zweiten Kapitel wird die für das Buch grundlegende Positionsbestimmung vorgenommen: Die beiden Autoren verstehen Medienbildung als Strukturale Medienbildung. Diese leiten sie aus einem Verständnis von Bildung her, das sie streng von einem materialen Bildungsbegriff abgrenzen. Ist Bildung in letzterem Verständnis als Ergebnis der Auseinandersetzung mit kanonischen Lern- und Ausbildungsinhalten zu verstehen (z.B. bestimmten Werken der Literatur), resultiert das strukturale Bildungsverständnis aus der Beobachtung von in der Moderne bzw. Postmoderne zunehmend entstehenden 
gesellschaftlichen Krisenzuständen, die sich für das Subjekt als Orientierungskrisen niederschlagen. Die Schlüsselprobleme unserer Gegenwart dazu gehört auch die große Dynamik der Einführung neuer Informationsund Kommunikationstechnologien - stellen das Individuum vor neue Handlungs- und Entscheidungsprobleme, die es nur dann angemessen lösen kann, wenn es über die nötige Flexibilität verfügt, sich gerade in Umbruchsituationen Orientierung zu verschaffen. Die Autoren zeigen auf, dass diese gleichsam strukturalen Elemente von Bildung nicht neu sind, sondern bereits in den Bildungsverständnissen Humboldts (1820, 1827-1829) und Klafkis $(1975,1985)$ aufscheinen. Neu sind freilich die gegenwärtigen gesellschaftlichen Zustände, die mit Verweis auf die Überlegungen von Giddens (1996) durch den Verlust sozialer Tradierungen und die gleichzeitige Zunahme eines Lebens mit höherer Kontingenz gekennzeichnet sind. Um sich als Subjekt in dieser durch Unbestimmtheit und Komplexität charakterisierten Gesellschaftskonstellation orientieren zu können, reicht die Stärkung von Verfügungswissen (Faktenwissen) nicht aus. Mehr als bisher muss der Einzelne ein besonderes $\mathrm{Ma} ß$ an reflexivem Orientierungswissen ausbilden. Es ist die Bildungstheorie, so Jörissen/Marotzki, die dazu prädestiniert ist, für dieses Aufgabenfeld den begrifflichen und konzeptionellen Rahmen zu liefern. Allerdings haben es bildungstheoretische Ansätze in der Vergangenheit verpasst, die Bedeutung von Medien ernst zu nehmen. Das von den Autoren vorgestellte Konzept von Medienbildung ändert diesen Zustand insofern, als es die Relevanz von Medien für Bildungs-, Subjektivierungs- und Orientierungsprozesse anerkennt, und zwar in zweifacher Hinsicht: Erstens sind Medien ein Bestandteil unserer Lebenswelt, zweitens bieten sie neue Anlässe und Räume für Bildungserfahrungen und -prozesse im Sinne orientierender Reflexion. Maßgebend für das vorgestellte Konzept von Medienbildung ist zudem, dass menschliche Ausdrucksformen - mit Verweis auf die Arbeiten von Jung (2005) bezeichnen sie diese als «Artikulationen» - nicht von Medialität zu trennen sind und dass mediale soziale Räume in den digitalen Medien eine zunehmend größere Rolle für Bildungsprozesse spielen.

Auf dieser theoretischen Basis ist es Aufgabe des zweiten Teils der Monografie (Kapitel 3 bis 5, S. 95-238), einerseits die reflexiven Potenziale von medialen Räumen zu erkennen, andererseits die vorfindbaren medialen Artikulationsformen anhand von vier Orientierungsdimensionen zu analysieren. Jörissen/Marotzki lehnen letztere an die vier, von Immanuel Kant (1800, 448 ) in seiner Logik formulierten Fragen an: 1) Was kann ich wissen? (Wis- 


\section{Rezensionen}

sensbezug), 2) Was soll ich tun? (Handlungsbezug), 3) Was darf ich hoffen? (Grenzbezug), 4) Was ist der Mensch? (Biografiebezug). Für ihre Analyse wählen die Autoren unterschiedliche Phänomene aus den Medien Film (Kapitel 3), Bild (Kapitel 4) und Internet (Kapitel 5) aus.

Film

Basierend auf der Form- und Strukturanalyse und seinen darauf entwickelten literarischen Russischen Formalismus leiten die Autoren im dritten Kapitel «Audiovisuelle Artikulationsformen» in die neoformalistische Filmanalyse ein. Beispielhaft anhand des Films «Ararat» (2002) von Atom Egoyan wird eine strukturale Filmanalyse durchgeführt sowie die Reflexionsoptionen durch Modalisierungen, der Wechsel des Wirklichkeitsmodus, und Diskursivität, die Bedeutungsmodifikation von Vergangenheit, des Films veranschaulicht. Anschließend werden die vier Bildungsdimensionen auf der Narrationsebene mittels historischer und aktueller, oft populärer Filme diskutiert. In einer abschließenden Vertiefung wird die Thematik des Filmpotentials hinsichtlich der Biographie- und Erinnerungsarbeit am Beispiel von Andrej Tarkowskijs «Der Spiegel» aufgegriffen.

\section{Bild}

Inspiriert durch das Filminterpretationsmodell von David Bordwell und Kristin Thompson (2008) entwickeln Jörisson und Marotzki in «visuelle Artikulationsformen» die Bildinterpretation von Erwin Panofsky (1962) weiter aus und untergliedern sie in vier Stufen. Die Autoren folgen dem Gedanken, «dass das Medium Film komplexere Strukturen aufweist, die jene Strukturen beinhalten, durch welche Bilder charakterisiert sind» (101), so dass der neoformalistische Ansatz und somit Elemente der Filmanalyse Einzug in die Bildinterpretation erhalten. Für jede Bildungsdimension werden nach der erweiterten Bildinterpretation jeweils zwei Bilder zuerst objektiv und im zweiten Schritt die Anordnung ihrer abgebildeten Objekte hinsichtlich ihrer Bedeutung und ihren Sinn beschrieben. In einem dritten Interpretationsschritt liegt der Fokus auf die Inszenierung der dargestellten Objekte angelehnt an dem oben genannten Filminterpretationsmodell, so dass das Bild in seiner Autonomie begriffen wird. Im letzten Schritt des Modells werden die Bilder bildungstheoretisch hinsichtlich ihrer Selbst- und Werterhaltung analysiert. 
Internet

Im letzten Hauptkapitel «Neue Artikulations- und Partizipationsräume des Internet» heben die Autoren sowohl die historische, als auch die aktuelle Entwicklung des Internets hervor und verweisen auf die gesellschaftliche Bedeutung bezüglich neuer Kulturräume. Exemplarisch stehen für die drei Bildungsdimensionen Wissensbezug, Handlungsbezug und Biographiebezug spezifische Web 2.0 Angebote: So wird zum Beispiel zur Bildungsdimension Wissensbezug nahe liegend Wikipedia als kollaborative Wissensprojekte und das Bloggen als neuen Artikulationsraum vorgestellt. Bezüglich des Handlungsbezuges stehen webbasierte soziale Netzwerke, kurz Communities, und für den Biographiebezug unter anderem Plattformen, welche kollektiv Geschichte erzählen wie zum Beispiel zeitzeugengeschichte.de, aber auch solche, die das Microblogging wie zum Beispiel twitter.com, anbieten. Die Cyborg-Thematik mit der Durchdringung von Körperlichkeit und Technologie in Form von Avataren stehen für den Grenzbezug.

Benjamin Jörissen und Winfried Marotzki ist es in ihrem Buch gelungen darauf hatten wir eingangs bereits aufmerksam gemacht -, einen im medienpädagogischen Diskurs lange erwarten systematischen Begründungsrahmen für Begriff und Konzept von Medienbildung vorzulegen. Mithilfe einer Fülle von medialen Beispielen versteht es das Werk zudem, die reflexiven Potenziale medialer Räume und medialer Artikulationsformen konkret zu machen. Dabei ist insbesondere Kapitel 5 («Neue Artikulations- und Partizipationsräume des Internet») hervorzuheben, in dem wichtige Phänomene und sozialisatorische Aspekte des Internet (etwa soziale Netzwerke, Avatare, Blogosphäre) anhand der vier Orientierungsdimensionen von Bildung analysiert werden. Die Autoren verstehen es, ihre komplexe Argumentation sprachlich verständlich darzustellen, so dass dieses Werk grundsätzlich nicht nur Wissenschaftlern, sondern auch Studierenden der Erziehungswissenschaft bzw. Medienpädagogik zu empfehlen ist.

Im Hinblick auf den Umfang der einzelnen Kapitel und ihre Gewichtung macht der mediale Analyseteil (Kapitel 3-5) den Schwerpunkt des Buches aus. Die bildungstheoretische Grundlegung (Kapitel 2) ist für eine Einführung in Medienbildung angemessen; angesichts der theoretischen Tiefe des Gegenstandes, hätte diese aber durchaus umfänglicher ausfallen dürfen. Vielleicht erklärt dies auch, warum die Autoren einige wichtige, nahe liegende Fragen offen lassen, allenfalls implizit berücksichtigen: 
Dazu gehört erstens die Frage nach dem Verhältnis von Medienbildung zu anderen wichtigen Begriffen und Konzepten der Medienpädagogik: Jörissen/ Marotzki argumentieren eng bildungstheoretisch. Die Beantwortung der Frage, wie der Einzelne mit den reflektierenden Formen seiner Wissensverarbeitung und -erzeugung umgeht, reservieren sie für die Bildungstheorie. Das ist konsequent, verdeckt aber, dass die Medienpädagogik in den letzten Jahren durchaus alternative Antworten zu finden versucht hat. In welchem Verhältnis also Medienbildung insbesondere zu Begriff und Konzept von Medienkompetenz steht, thematisieren die Autoren nur implizit, wenn sie Kritik an der gesellschaftlichen Akkumulation von Verfügungswissen üben. Aus bildungstheoretischer Perspektive wird in den letzten Jahren gerade Medienkompetenz verdächtigt, diesem Verfügungs- oder Faktenwissen zu nahe zu stehen. Die Kritik freilich, Medienkompetenz habe einen vor allem zweckrationalen Charakter und könne zu leicht außerhalb der Selbstbestimmung des Subjekts dienenden Zielen untergeordnet werden, greift unseres Erachtens zu kurz, weil sie sich in erster Linie auf bestimmte Verwendungsweisen des Begriffs (z.B. in ökonomischen Zusammenhängen) bezieht. Die Erklärungskraft von Medienkompetenz zeigt sich darin, dass jeder Mensch ein kompetentes Wesen ist und deshalb als dazu in der Lage zu betrachteten ist, grundsätzlich auch kompetent mit Medien handeln zu können. Und: Betrachtet man Medienkompetenz (z.B. in der Lesart von Dieter Baacke: Medienkritik, Medienkunde, Mediennutzung und Mediengestaltung) bzw. seine theoretische Herleitung (Kommunikative Kompetenz) begrifflich präzise, zeigt sich, dass auch in Medienkompetenz eine reflexive Komponente mitgedacht und auch ausformuliert ist, und zwar als Selbstkompetenz, personale Kompetenz oder Medienkritik. Vor diesem Hintergrund scheint es kaum plausibel zu sein, Medienbildung anstelle von Medienkompetenz zu verwenden. Nahe liegender ist es, beide Begriffe und Konzepte integrativ zu betrachten - wer von dem einen redet, muss das andere mit einbeziehen. Wer in dem Buch von Jörissen/Marotzki Hinweise auf diese Zusammenhänge bzw. auf die Positionierung von Medienbildung im Verhältnis zu jenseits der Bildungstheorie angesiedelten medienpädagogischen Schlüsselbegriffen und -konzepten sucht, wird kaum fündig.

Eine zweite Frage, die das Buch offen lässt, ist das Verhältnis von Medienbildung und beruflicher bzw. professioneller Praxis der Medienpädagogik. Den Autoren geht es um die theoretische Herleitung des Begriffs und um die Analyse der Bildungsprozesse, die in und mithilfe der Medien stattfinden. Derjenige Leser wird enttäuscht werden, der sich in dem Buch Schlussfolge- 


\section{Rezensionen}

rungen für konkretes (medien-)pädagogisches Handeln mit Kindern, Jugendlichen oder Erwachsenen erwartet. In diesem Zusammenhang bleibt es auch insgesamt abzuwarten, wie sich Medienbildung, als ein ernsthaft verwendeter Zielwert für Medienpädagogik in Theorie und Praxis durchsetzen wird.

Man ist beim Lesen des Werkes versucht, die Autoren einmal selbst nach ihren Einschätzungen hinsichtlich dieser und anderer Aspekte zu befragen. Benjamin Jörissen und Winfried Marotzki gehen an dieser Stelle einen innovativen Weg, indem Sie parallel zum Erscheinungstermin die Internetseite

\section{http://www.strukturale-medienbildung.de}

freigeschaltet haben, auf der (nicht nur) die Leser des Buches u.a. über wichtige Grundbegriffe (Glossar), Links und best practice-Beispiele studentischer Projekte zum Gegenstandes informiert werden sowie die Kontaktaufnahme mit den Autoren etwa über Facebook suchen können.

Kai-Uwe Hugger/llona Cwielong

\section{Literatur}

Bordwell, David. Thompson, Kristin: Film art. An introduction. (8., internat. ed.). Boston: McGraw Hill Higher Education, 2008.

Giddens, Anthony. Konsequenzen der Moderne. Frankfurt/M: Suhrkamp, 1996.

Humboldt, Wilhelm von. Über das vergleichende Sprachstudium in Beziehung auf die verschiedenen Epochen der Sprachentwicklung. In: Ders.: Werke in fünf Bänden. Bd. 3. (3. Aufl.). Darmstadt: Wissenschaftl. Buchgesellschaft, 1820; 1980, S. 1-25.

Humboldt, Wilhelm von. Über die Verschiedenheiten des menschlichen Sprachbaus. In: Ders.: Werke in fünf Bänden. Bd. 3. (Aufl.). Darmstadt: Beltz, 1827-1829; 1980, S. 144-367.

Klafki, Wolfgang. Studien zur Bildungstheorie und Didaktik. Weinheim/Basel: 


\section{Rezensionen}

Beltz, 1975.

Klafki, Wolfgang (Hrsg.). Neue Studien zur Bildungstheorie und Didaktik. Beiträge zur kritisch-konstruktiven Didaktik. Weinheim/Basel: Beltz, 1985.

Panofsky, Erwin: Studien zur Ikonologie. In: Studien zur Ikonologie. Humanistische Themen in der Kunst der Renaissance. Köln: Dumont 1962; 1980, S.29-61. 